\title{
Resource Aware Gaussian Regressive Jarvis Patrick Clustering for Reliable Data Transmission in E-Health using WSN-IoT
}

\author{
M. Suguna1, Dr. S. Sathiyabama ${ }^{2}$, Dr. R. Reka $^{3}$ \& Dr. K. V. Umashankar ${ }^{4}$ \\ ${ }^{1}$ Research Scholar, Bharathiyar University, Coimbatore-641046, Tamilnadu, India. \\ Department of Computer Science, Vinayaka Mission's Kirupananda Variyar Arts and Science \\ College, Salem-636308, Tamilnadu, India. \\ ${ }^{2}$ Assistant Professor, Department of Computer Science, Thiruvalluvar Government Arts College, \\ Rasipuram-637401, Namakkal, Tamilnadu, India. \\ ${ }^{3}$ Professor \& Head, Department of Computer Science \& Engineering, Annai Mathammal Sheela \\ Engineering College, Erumapatty, Namakkal - 637013, Tamilnadu, India. \\ ${ }^{4}$ Assistant Professor, Department of Computer Science, Shanmuga Industries Arts and Science \\ College, Tiruvannamalai-606603, Tamilnadu, India. \\ 1'sugunam.2007@gmail.com, ${ }^{2}$ sathyaaksrct@yahoo.com, ${ }^{3}$ rekasatheesh@gmail.com and \\ 4sukar1317@gmail.com
}

Article History: Received: 10 November 2020; Revised 12 January 2021 Accepted: 27 January 2021; Published online: 5 April 2021

\begin{abstract}
Wireless sensor network (WSN) includes numerous sensor nodes ( $\mathrm{SN}$ ) integrated with Internet of Things (IoT) play a crucial role in numerous applications. IoT links physical devices as sensor and forms whole network for sharing information. The IoT has been used in different domains. In this scenario, patients utilize wearable medical sensors to monitor medical parameters. This medical sensor is equipped with batteries and has limited energy. Therefore, the network lifetime enhancement is a major challenging issue. To prolong network lifetime, novel technique called Resource-efficient Gaussian process regressive Jarvis Patrick clustering (REGPRJPC) is introduced. At first, the IoT devices are used in $\mathrm{SN}$ for sensing and collecting the patient data. After data collection process, $\mathrm{SN}$ is grouped into diverse clusters using Jarvis Patrick clustering technique. Jarvis Patrick clustering is graphbased clustering to partition SN with help of Gaussian process regression function. The regression function analyzes the SN and performs the clustering process based on the estimated energy and bandwidth. After clustering process, cluster head $(\mathrm{CH})$ is selected to enhance data transmission and minimizes delay. Source node transmits gathered data to their $\mathrm{CH}$. Then $\mathrm{CH}$ finds nearest $\mathrm{CH}$ using time of flight method. Followed by, data transmission is performed from source to sink node via the cluster head. In this way, resource-efficient data transmission is performed in WSN. Numerical analysis indicates that the REGPRJPC technique efficiently improves the reliable patient data packet delivery and minimizes the loss rate, delay.
\end{abstract}

Keywords: WSN, IoT, data transmission, Jarvis Patrick clustering, Gaussian process regression, time of flight. 


\section{Introduction}

WSN contains SN incorporated with IoT. IoT links physical devices to build network for exchanging information. In monitoring WSN with IoT, deployed SN is responsible to sense and observe data from environmental conditions and send to Base Station (BS). Network ensures data loss which leads to wastage of bandwidth and long-distance transmission outcomes in energy reduction. SN has restrictions in energy because of limited batteries. Therefore, clustering is common method for efficient transmission in WSN.

A Priority-Based Energy-Efficient Routing Protocol (PEERP) was presented in [1] to enhance reliability of data transmission. However, the data transmission delay was not minimized during the healthcare data transmission. An efficient data transfer mechanism was introduced in [2] to extend the network lifetime. But mechanism failed to sufficiently reduce the energy utilizing. A Fog-assisted Computational efficient Wearable sensor network (FCE-WSN) was developed in [3] for health monitoring. However, higher data delivery was not achieved. A high-quality clustering and $\mathrm{CH}$ selection were introduced in [4] using fuzzy logic for minimizing the total energy. But the algorithm failed to work with the higher density of nodes.

A CH selection algorithm was introduced in [5] to minimize the significant amount of energy utilization. However, delivery ratio performance was not improved. An energy-aware clusterbased routing protocol [6] enhance packet delivery ratio. However, delay was not minimized. The dynamic clustering-based methodology was proposed in [7] to enhance the data packet transmission. Designed method increases the throughput but the loss rate was not minimized.

A Scalable and energy-efficient routing protocol (SEEP) was designed in [8] to minimize energy consumption. However, the designed protocol failed to achieve higher data delivery with minimum delay. A novel routing protocol was presented in [9] to enhance network lifetime and lessen energy consumption. But, cluster-based routing was not applied to minimize the delay. The various energy-efficient techniques were developed in [10] for IoT-based heterogeneous wireless nodes. But the designed techniques failed to use multiple resources for data transmission.

\subsection{Contribution of the Proposed Work}

To resolve above-said issues, a novel REGPRJPC technique is introduced. The proposed REGPRJPC technique highlights the following primary contributions.

- To improve reliable data transmission, a REGPRJPC technique is developed based on energy and bandwidth consumption. This contribution is achieved based on Jarvis Patrick's clustering and Gaussian process regression.

- To enhance data delivery and lessen packet loss, Gaussian process regressive Jarvis Patrick clustering is introduced. At first, the IoT devices are used in SN to collect the patient data. SN is divided depends on energy and bandwidth. Jarvis Patrick clustering uses the Gaussian process regression to improve the clustering accuracy.

- To minimize the delay, the REGPRJPC technique uses time of flight method to choose neighboring $\mathrm{CH}$ for efficient transmission. 
- Finally, simulation was performed to compare REGPRJPC technique with conventional methods based on different metrics.

\subsection{Organization of paper}

The remaining sections of paper are arranged into various sections as below: Here, section 2 provides literature in energy-efficient data transmission in WSN. Section 3 offers architecture of REGPRJPC technique and described in detail. In Section 4, simulations are performed with the medical dataset. In Section 5, the performance of proposed and existing methods is presented with diverse metrics. Section 5 concludes the article.

\section{Related Works}

A novel method called Small world WSN (SW-WSN) was presented in [11] for data transmission. Designed method increases network lifetime but the packet loss rate was not minimized. A novel energy supply strategy was introduced in [12] for stable sensor data transmission. But the delay was not minimized during the data transmission.

An energy-aware emergency transmission method was introduced in [13] for reliable data transmission and minimizing the delay. The designed method failed to achieve reliable data transmission. A modified gravitational search algorithm was designed in [14] for Energyefficient clustering. Algorithm efficiently reduces the delay.

An improved dynamic hierarchical clustering technique was introduced in [15] to find the $\mathrm{CH}$ based on residual energy. But the higher data delivery rate was not minimized. A multiObjective Based Clustering and Sailfish Optimizer (SFO) was introduced in [16]. The delay was not reduced.

Fitness based Glowworm swarm with Fruitfly algorithm was designed in [17] to discover $\mathrm{CH}$ and enhance network lifetime. But the designed algorithm failed to use the clustering concept for minimizing the delay. An enhanced clustering hierarchy $(\mathrm{ECH})$ technique [18] to attain energy efficiency based on neighboring nodes. However, data delivery was not improved.

A multi-objective integer problem (MOIP) technique was introduced in [19] to optimize energy consumption and greater delivery. But technique failed to achieve the higher data delivery ratio with minimum delay. A queen honey bee migration (QHBM) was introduced in [20] to minimize the delay of data transmission. The resource-efficient data transmission remained unsolved.

\section{Methodology}

In recent days, the technologies of WSN are applied in various fields due to their low cost and simple operation. In WSN, numerous SN are distributed in observing the field to sense the required data. All of these data are collected and send towards the BS via single or multi-hop adopted data transmission model.

IoT incorporates to WSN that connects a large sensor devices using internet to gather data. IoT-based WSN includes different domains such as agriculture, industry, smart health, surveillance, and so on. The IoT-based WSN has been applied to the E-health monitoring 
system to minimize the time and human efforts, and increase the data transmission in a quality manner.

To prolong the networks lifetime and lessen energy consumption in Healthcare using IoT, an efficient REGPRJPC is introduced to send data from SN to sink node.

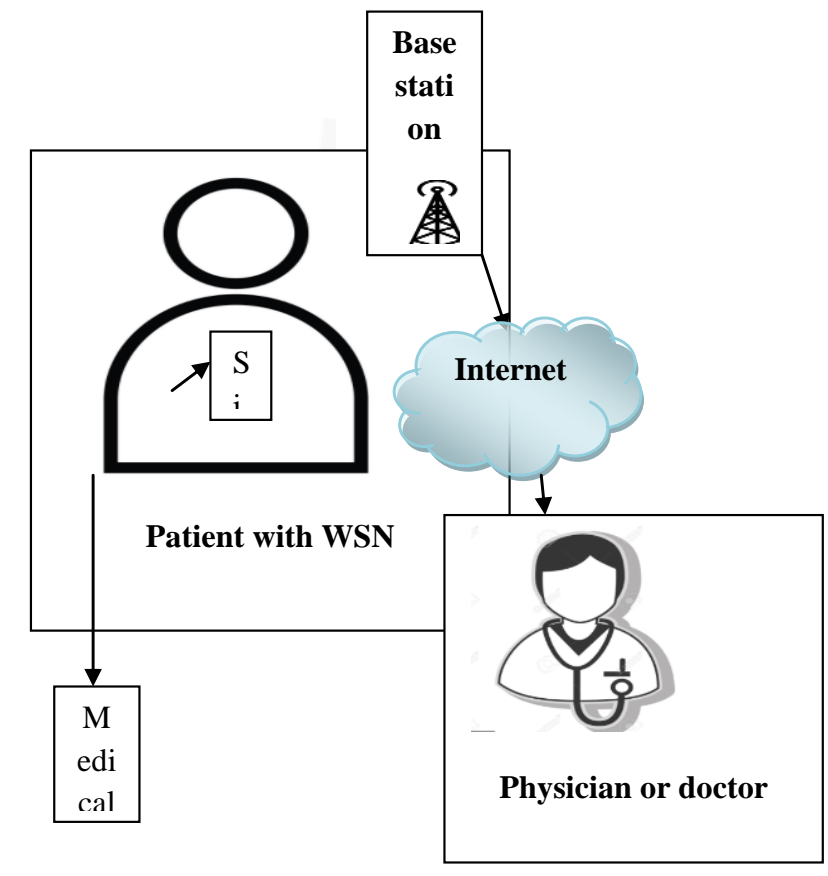

Figure 1. IoT-based E-health monitoring system architecture

Figure 1 portrays E-health care monitoring system with IoT-based WSN. Sensors are positioned in surface of patient body to observe various signs such as glucose level, blood pressure, temperature and heart rates, and so on. Collected health information is sent to local processing unit called sink that transmits to hospital or healthcare system through internet for storage and data analysis. Gathered and stored medical data is accessible anytime and anywhere. During the data transmission, the energy is the significant metric to extend the overall lifetime of networks. To improve the data transmission in E-Healthcare with IoT, novel REGPRJPC technique is introduced.

\subsection{Network model}

Consider WSN has arranged undirected graph ' $G=(V, E)$ ', where ' $V$ ' denotes medical sensor nodes $S n_{i} \in S n_{1}, S n_{2}, S n_{3}, \ldots . S n_{n}$ deployed in a square area $n * n$ and ' $E$ ' indicates the edges i.e. link between the nodes. The source node $(S N)$ transmits a patient data i.e. data packet $p_{1}, p_{2}, p_{3}, \ldots p_{n}$ to sink node ' $S$ ' through the resource-efficient nodes with better link quality. Based on the above network model, REGPRJPC is designed 


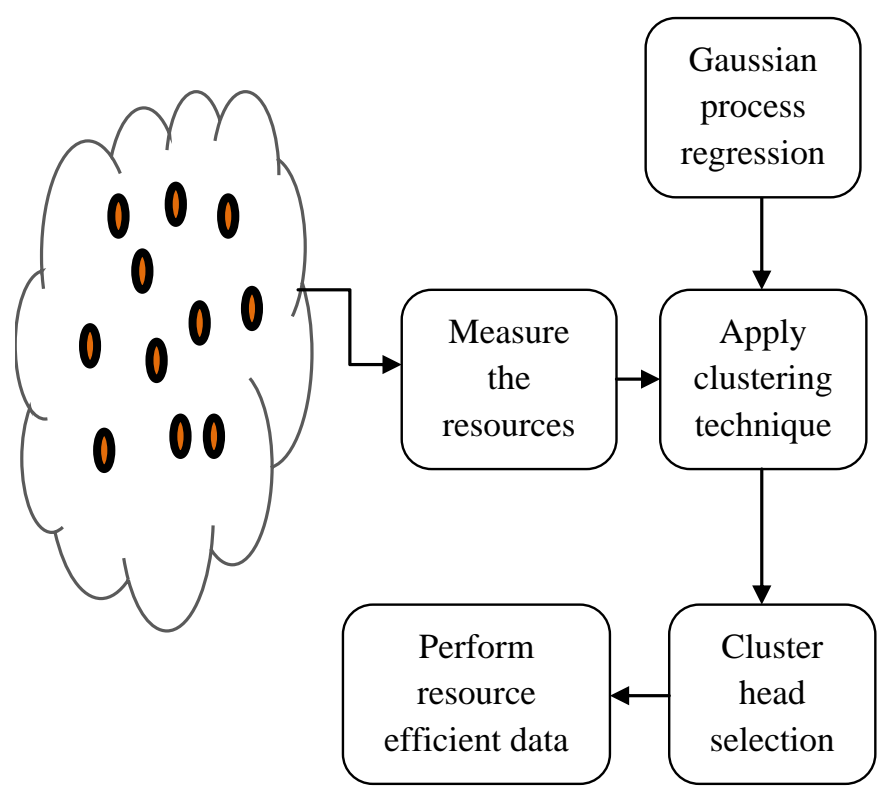

Figure 2. Block diagram of the proposed REGPRJPC technique

Fig 2 illustrates a diagram of REGPRJPC to obtain higher data delivery. For each medical SN, different resources such as energy and bandwidth are measured. With resource estimation, the $\mathrm{SN}$ are clustered using the Gaussian process regressive Jarvis Patrick graph clustering technique. After the clustering, the $\mathrm{CH}$ is selected to send patient data. Then $\mathrm{CH}$ transmits patient data to sink node through neighboring $\mathrm{CH}$. As a result, resource-optimized data transmission is performed.

\subsubsection{Gaussian process regressive Jarvis Patrick graph clustering}

The proposed REGPRJPC technique uses the Jarvis Patrick clustering to partition the SN to find the resource-efficient nodes for reliable data transmission. The Jarvis Patrick clustering is the graph-based clustering technique to improve data transmission and minimizes delay. Cluster-based data transmission is the efficient approach in which the head nodes collect data from each nodes and then sends it to sink node for further processing. This process improves $t$ higher data delivery and reduces delay. Besides, resource-efficient clustering process is also used to prolong the network lifetime.

Consider undirected graph ' $G=(V, E)$ ', where ' $V$ ' indicates the medical sensor nodes $S n_{i} \in$ $S n_{1}, S n_{2}, S n_{3}, \ldots . S n_{n}$ deployed in a square area $n * n$ and ' $E$ ' indicates the edges i.e. link between the nodes.

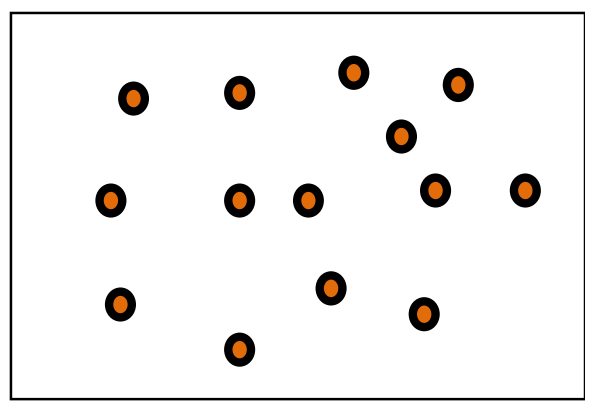

Figure 3. deployments of sensor nodes in the square area 
Figure 3 portrays SN deployment in square area and starts to sense and monitor remote patient activities in E-health application. Jarvis Patrick's clustering worked based on nearest neighbor concept for every object in set to be clustered. On the contrary to the conventional clusterbased routing technique, the proposed REGPRJPC technique uses the Gaussian process regression to improve the clustering performance and minimizes the error that occurred during the clustering process.

For each SN, energy and memory consumption are measured to find resource-efficient node to improve network lifetime. Each SN has same energy level. SN Energy is calculated depends on product of power and time is expressed as follows,

$$
E_{S n}=[\text { power }] *[\text { time }]
$$

Where, ' $E_{S n}$ ' symbolizes the energy and is measured in the unit of joule $(\mathrm{J})$. Due to sensing and monitoring quality of $\mathrm{SN}$, energy level gets degraded therefore remaining energy (i.e. residual energy) is measured as below,

$$
R_{E_{S n}}=E_{S n_{t}}-E_{S n_{c}}
$$

Where, $R_{E_{S n}}$ indicates the residual energy, $E_{S n_{t}}$ indicates total energy, $E_{S n_{c}}$ be the energy consumed by the SN. Followed by, the bandwidth consumption is measured as the difference between the total bandwidth and available bandwidth of the SN which is expressed a given below,

$$
B_{C}=\left[B_{T}\right]-\left[B_{a}\right]
$$

Where, $B_{C}$ indicates the bandwidth consumption, $B_{T}$ indicates a total bandwidth, $B_{a}$ denotes the available bandwidth. Based on estimated resources, the clustering process is carried out to group the SNs.

By applying the Jarvis Patrick clustering technique, the SN are clustered based on the nearest neighbor concept. The Gaussian process regression is applied for grouping the SNs. Gaussian process regression is a machine learning technique that helps to find the relationship (i.e. similarity) between the SN based on their resources. Initially, the numbers of clusters are randomly initialized.

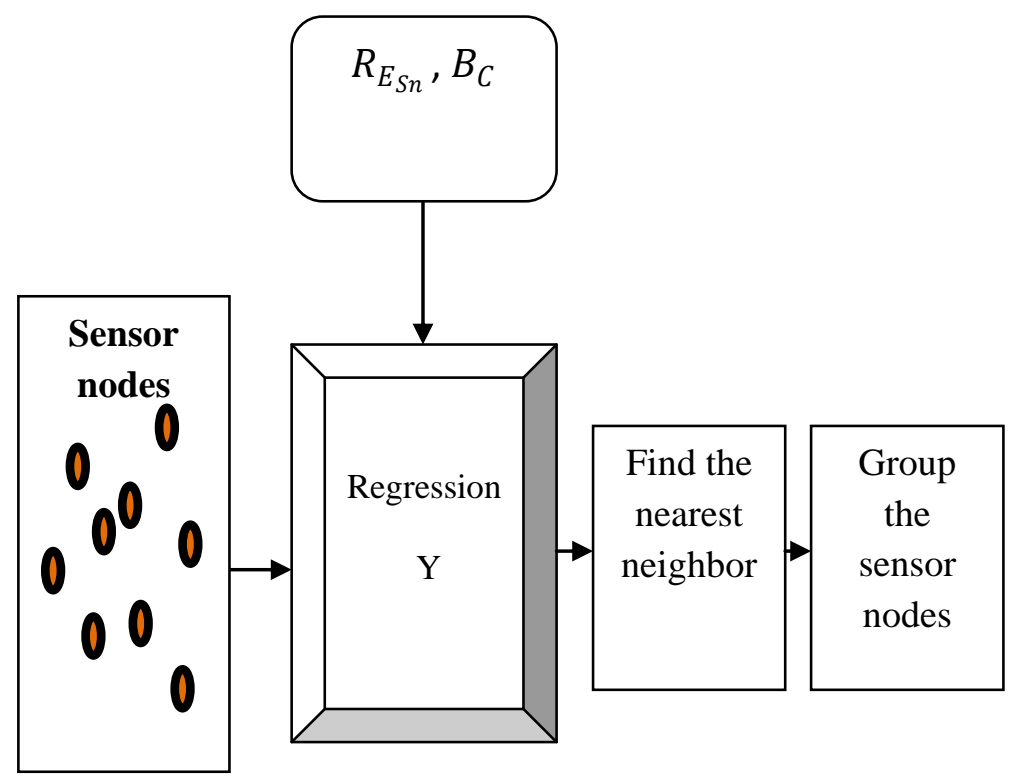

Figure 4. Gaussian process regressive Jarvis Patrick graph clustering 
Figure 4 demonstrates process of Gaussian process regressive Jarvis Patrick graph clustering technique. Number of $\mathrm{SN}$ and estimated resources is given to regression function to find the nearest neighbors.

$$
Y=\exp \left(\frac{\left|S n_{i}-S n_{j}\right|}{2 d^{2}}\right)
$$

Where, $Y$ denotes a regression output, $S n_{i}, S n_{j}$ denotes a sensor node, ' $d$ ' indicates a deviation, $\left|S n_{i}-S n_{j}\right|$ indicates distance among two SNs. Minimum distance between nodes is grouped into the particular cluster. In other words, the SN that have similar resource level are grouped into the particular cluster. The regression function provides the output ranges from 0 to 1 .

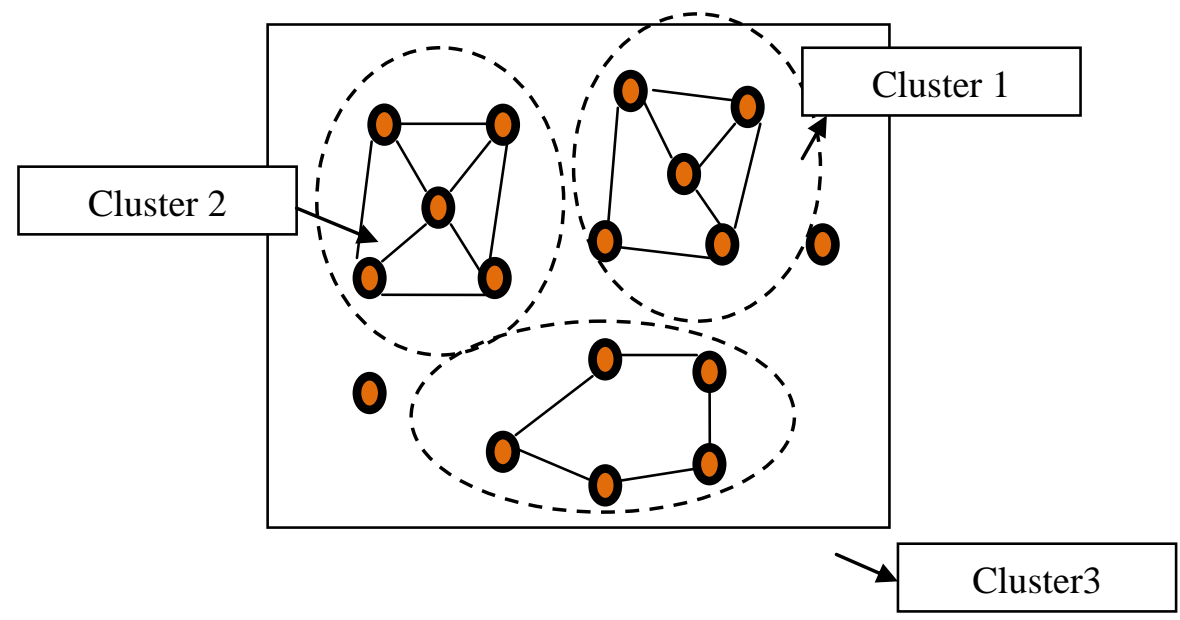

Figure 5. Sensor node clustering

Figure 5 shows the clustering of SN based on similarity among nodes. Nodes with higher similarity are clustered to particular cluster based on undirected graphical models. Each SN is clustered into particular cluster. After clustering process, $\mathrm{CH}$ is chosen for efficient transmission as well as enhances network lifetime. For each cluster, the node with greater residual energy and bandwidth consumption is selected for reliable data transmission.

$$
F=\left[\arg \max \left(R_{E_{S n}}\right) \& \& \arg \min \left(B_{C}\right)\right]
$$

Where, $F$ denotes a function to verify the $\mathrm{SN}$ resources such as residual energy $\left(R_{E_{S n}}\right)$ and bandwidth consumption $\left(B_{C}\right), B_{C}$. After choosing $\mathrm{CH}$, source node sends patient data into their cluster head. Then the $\mathrm{CH}$ discovers neighboring $\mathrm{CH}$ to send data to sink node. The nearest $\mathrm{CH}$ is determined depends on time of flight method. $\mathrm{CH}$ transmits beacon message to other $\mathrm{CH}$. Based on arrival time of beacon message, neighboring $\mathrm{CH}$ is selected. Time of flight $(A)$ is measured as dissimilarity among beacon message arrival time and beacon message transmission time. It is mathematically expressed as given below,

$A=$ message arrival time - message transmission time (6)

The nearest $\mathrm{CH}$ is chosen depends on beacon message arrival from neighboring $\mathrm{CH}$. After that, $\mathrm{CH}$ sent patient data into neighboring $\mathrm{CH}$. In this way, reliable data transmission is performed from source to sink node with minimum delay. 


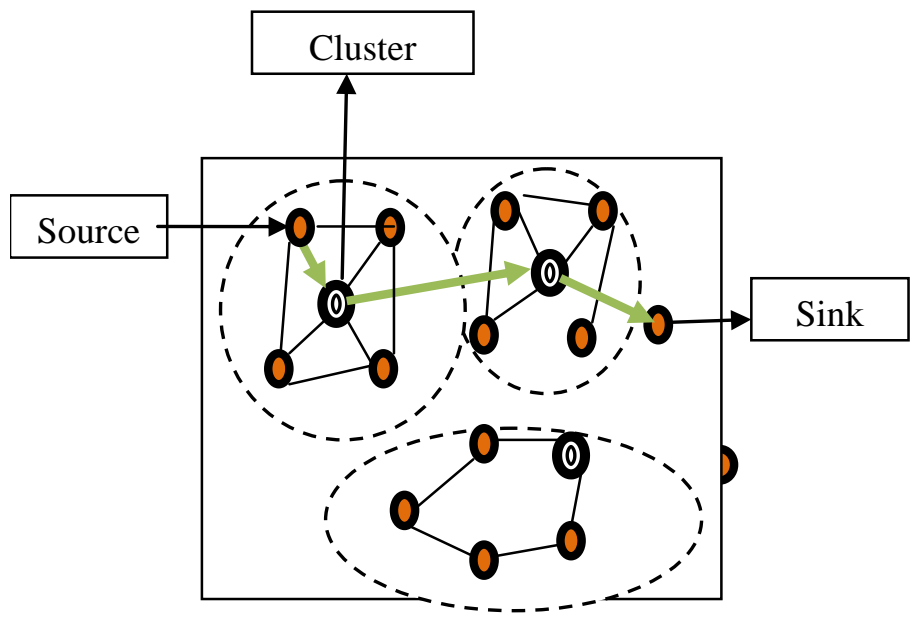

Figure 6. clustering-based data transmission

Figure 6 illustrates the clustering-based data transmission is performed between source to sink node. $\mathrm{SN}$ has the data, send to $\mathrm{CH}$ and $\mathrm{CH}$ transmits to sink.

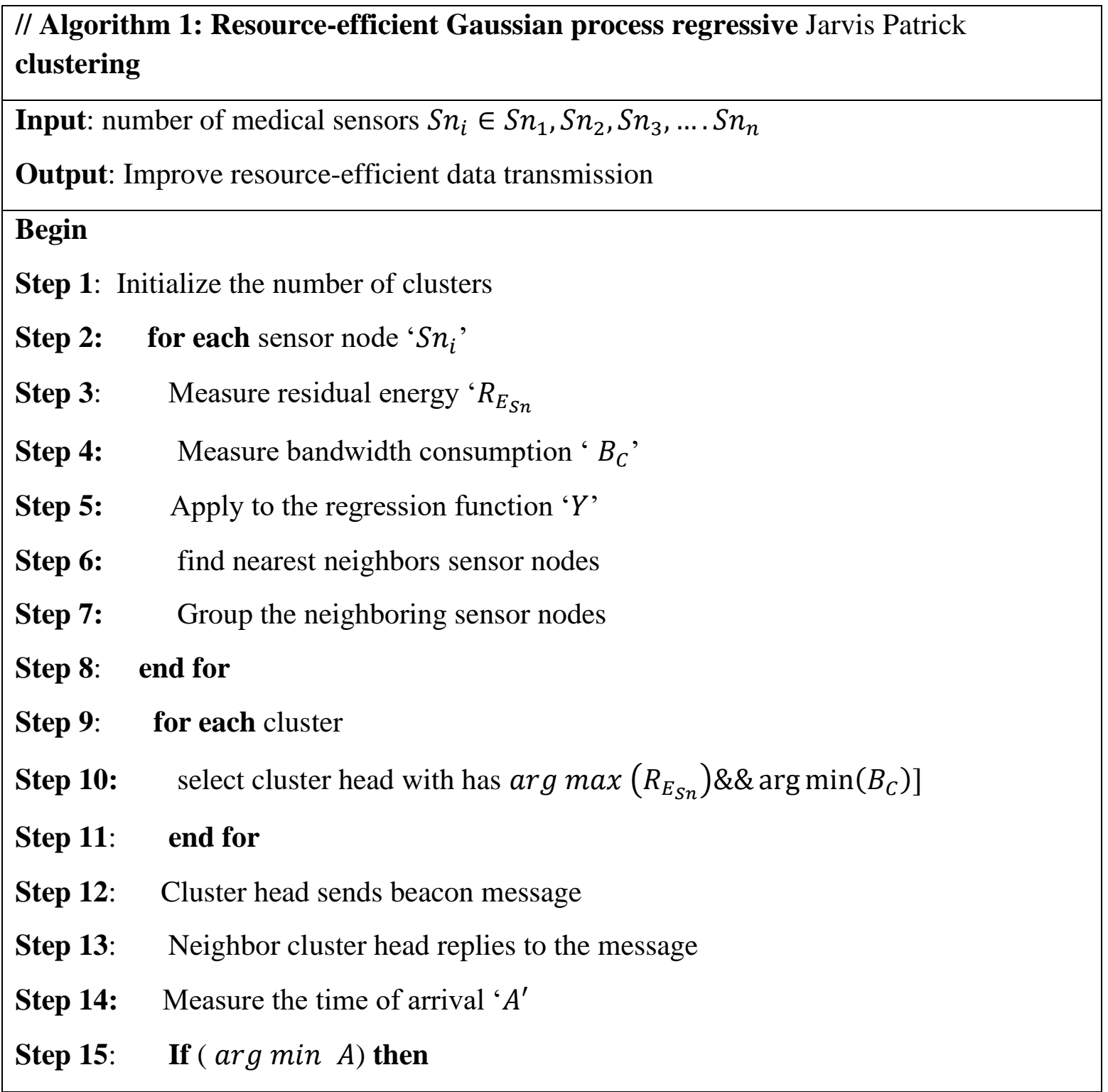




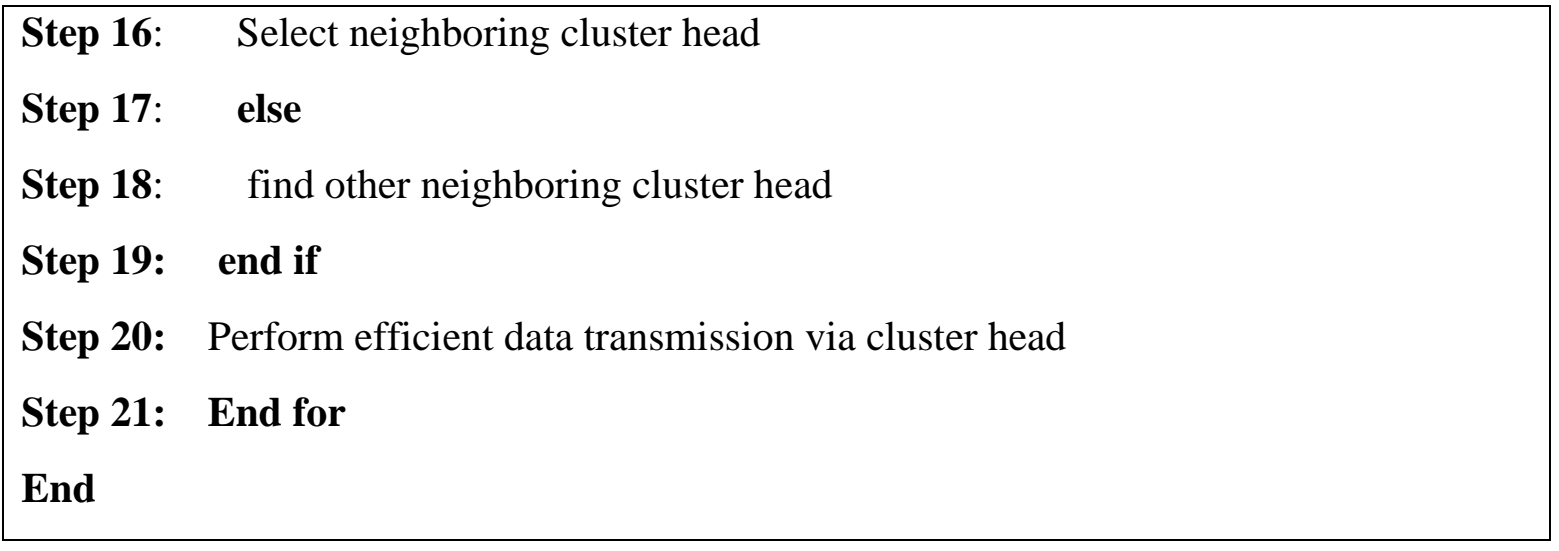

Algorithm 1 describes process of REGPRJPC to perform resource-efficient data transmission in WSN. Initially, the number of clusters is randomly initialized. For each SN, residual energy and bandwidth consumption are calculated. Then the Gaussian process regression is applied for analyzing the resources of the SNs. The nodes that have higher similarity are grouped into the particular cluster. After clustering the $\mathrm{SNs}$, the $\mathrm{CH}$ is chosen depends on higher residual energy and lesser bandwidth consumption. Source node transmits gathered patient data into their $\mathrm{CH}$. Followed by, $\mathrm{CH}$ finds the neighboring $\mathrm{CH}$ based on the time of flight method. Finally, reliable data transmission is performed between the sources and sink node via a resource-efficient $\mathrm{CH}$. This increases data transmission and reduces delay.

\section{Simulation Settings}

Simulations are implemented in NS2.34 simulator for REGPRJPC technique and existing PEERP [1] and efficient data transfer mechanism [2]. To make a fair comparison between the existing and proposed methods, the environmental setup for conducting simulations are performed by positioning $500 \mathrm{SN}$ dispensed in squared area of $A^{2}(1100 \mathrm{~m} * 1100 \mathrm{~m})$. Patient data are collected from the MHEALTH Dataset

[http://archive.ics.uci.edu/ml/datasets/mhealth+dataset].

MHEALTH (Mobile Health) dataset is used for monitoring the patient activities based on multimodal body sensing using sensors placed on patient's chest, right wrist, and left ankle. Sensor located in chest also offers 2-lead ECG patterns employed for basic heart monitoring, checking for a variety of exercise on ECG patient. Dataset includes body activities and signs recordings for ECG patients when performing 12 physical activities. Collected body activities from $\mathrm{SN}$ are transmitted to $\mathrm{BS}$ via internet to further processing.

During the data transmission, Random Waypoint model is employed as mobility model used in simulation scenario to perform resource-efficient data transmission in WSN. The simulation time is 300 seconds with movement node speed of $0-20 \mathrm{~m} / \mathrm{sec}$. Besides, data packet varies from 50 and 150. 
Table 1. Simulation Parameters

\begin{tabular}{|l|l|}
\hline $\begin{array}{l}\text { Simulation } \\
\text { parameter }\end{array}$ & \multicolumn{1}{|c|}{ Value } \\
\hline Simulator & NS2 .34 \\
\hline Network area & $1500 \mathrm{~m} * 1500 \mathrm{~m}$ \\
\hline $\begin{array}{l}\text { Number of } \\
\text { mobile nodes }\end{array}$ & \begin{tabular}{l}
$50,100,150,200,250,300,350,400$ \\
\hline Protocol
\end{tabular} \\
\hline Simulation time & $300 \mathrm{sec}$ \\
\hline Mobility model & Random Way Point model \\
\hline Nodes speed & $0-20 \mathrm{~m} / \mathrm{s}$ \\
\hline Number of runs & 10 \\
\hline
\end{tabular}

\section{Results And Discussion}

Simulation results of REGPRJPC and PEERP [1] and efficient data transfer mechanism [2] are discussed with different performance metrics. Performance results are discussed with assist of table or graphical representation.

\section{a. Impact of packet delivery ratio}

Packet delivery ratio is measured as ratio of number of patient data i.e. data packets are successfully delivered at destination to total number of data packets. Packet delivery ratio is formulated as below,

$$
P D_{R}=\left(\frac{D P R}{D P S}\right) * 100
$$

In (7), $P D_{R}$ indicates packet delivery ratio, $D P R$ represents data packet received, $D P S$ indicates data packet sent. Packet delivery ratio is calculated in percentage (\%).

Table 2. Tabulation for Packet delivery ratio

\begin{tabular}{|c|c|c|c|}
\hline \multirow{2}{*}{$\begin{array}{c}\text { Number of } \\
\text { sensor nodes }\end{array}$} & \multicolumn{3}{|c|}{ Packet delivery ratio (\%) } \\
\cline { 2 - 4 } & REGPRJPC & PEERP & $\begin{array}{c}\text { Efficient data } \\
\text { transfer mechanism }\end{array}$ \\
\hline $\mathbf{5 0}$ & 87 & 77 & 73 \\
\hline $\mathbf{1 0 0}$ & 88 & 82 & 78 \\
\hline $\mathbf{1 5 0}$ & 91 & 87 & 84 \\
\hline $\mathbf{2 0 0}$ & 90 & 88 & 85 \\
\hline $\mathbf{2 5 0}$ & 92 & 87 & 84 \\
\hline $\mathbf{3 0 0}$ & 89 & 86 & 83 \\
\hline $\mathbf{3 5 0}$ & 90 & 85 & 82 \\
\hline $\mathbf{4 0 0}$ & 89 & 88 & 86 \\
\hline
\end{tabular}




\begin{tabular}{|l|l|l|l|}
\hline $\mathbf{4 5 0}$ & 90 & 86 & 85 \\
\hline $\mathbf{5 0 0}$ & 88 & 85 & 83 \\
\hline
\end{tabular}

Performance analysis of packet delivery ratio is reported in table 2. Let us consider $50 \mathrm{SN}$ are 100 to conduct the simulation. By applying REGPRJPC, 87 data packets are successfully delivered to sink. Therefore, percentage of packet delivery ratio is $87 \%$. By applying existing methods PEERP [1] and efficient data transfer mechanism [2], the 77 and 73 data packets are successfully delivered at the destination and delivery ratios are $77 \%$ and $73 \%$. Similarly, nine remaining runs are performed for different methods. Totally, ten runs are performed for each method, and results are compared. The comparison results indicate that packet delivery ratio of REGPRJPC is increased by $5 \%$ and $9 \%$ when compared to conventional methods.

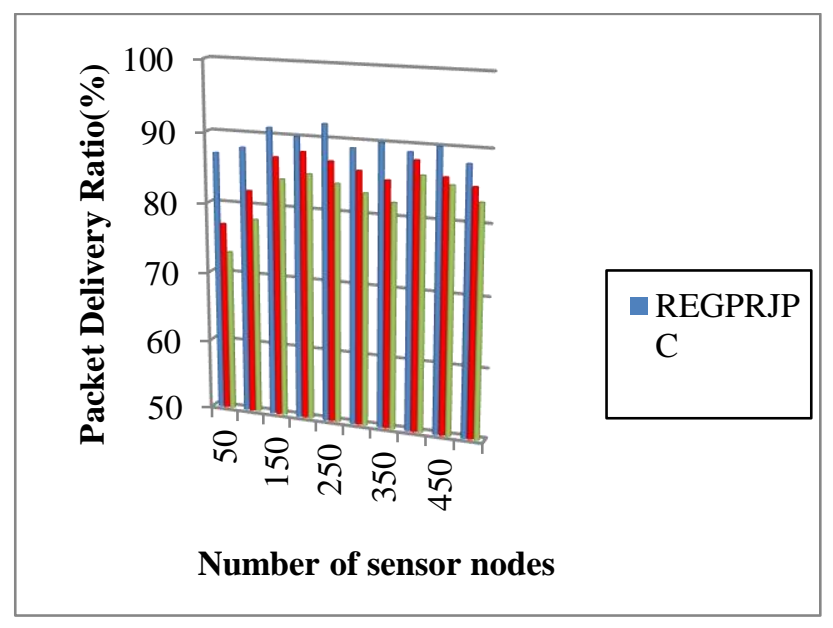

Figure 7. performance analysis of packet delivery ratio

Figure 7 illustrates results of packet delivery ratio. Packet delivery ratio of the different methods REGPRJPC technique and existing PEERP [1] and efficient data transfer mechanism [2] are represented by the three different colors such as blue, red, and green respectively. From figure 4, packet delivery ratio of REGPRJPC technique is enhanced. This improvement of the proposed REGPRJPC technique is to apply the Gaussian process regressive Jarvis Patrick graph clustering to find the resource-efficient SNs. By applying regression function to the clustering technique, the relationship between $\mathrm{SN}$ is measured depends on energy and bandwidth consumption. $\mathrm{CH}$ selection of REGPRJPC technique increases the data delivery from source to sink node.

\section{b. Impact of packet loss rate}

Packet loss rate is calculated as ratio of number of patient data i.e. data packets lost at the destination to total number of data packets sent. Data packet delivery ratio is expressed as given below,

$$
P L_{R}=\left(\frac{D L R}{D P S}\right) * 100
$$

Where, $P L_{R}$ denotes a packet loss rate, $D L R$ represents data packet lost, DPS indicates data packet sent. It is measured in percentage $(\%)$. 
Table 3. Tabulation for packet loss rate

\begin{tabular}{|c|c|c|c|}
\hline $\begin{array}{c}\text { Number } \\
\text { of sensor } \\
\text { nodes }\end{array}$ & \multicolumn{3}{|c|}{ Packet loss rate (\%) } \\
\cline { 2 - 4 } & REGPRJPC & PEERP & $\begin{array}{c}\text { Efficient data } \\
\text { transfer } \\
\text { mechanism }\end{array}$ \\
\hline $\mathbf{5 0}$ & 13 & 23 & 27 \\
\hline $\mathbf{1 0 0}$ & 12 & 18 & 22 \\
\hline $\mathbf{1 5 0}$ & 9 & 13 & 16 \\
\hline $\mathbf{2 0 0}$ & 10 & 12 & 15 \\
\hline $\mathbf{2 5 0}$ & 8 & 13 & 16 \\
\hline $\mathbf{3 0 0}$ & 11 & 14 & 17 \\
\hline $\mathbf{3 5 0}$ & 10 & 15 & 18 \\
\hline $\mathbf{4 0 0}$ & 11 & 13 & 14 \\
\hline $\mathbf{4 5 0}$ & 10 & 14 & 15 \\
\hline $\mathbf{5 0 0}$ & 12 & 15 & 17 \\
\hline
\end{tabular}

Table 2 given above describes the packet loss rate. The tabulated results indicate that the packet loss rate of the proposed REGPRJPC technique is reduced when compared to the conventional methods. This is proved through statistical analysis. Let us consider the $50 \mathrm{SN}$ and 100 patient data being sent from source node. Loss rate is $13 \%$ using the REGPRJPC technique whereas the loss rates of exiting methods PEERP [1] and efficient data transfer mechanism [2] are 23\% and $27 \%$ respectively. From the statistical analysis, results of proposed technique minimizes packet loss rate during patient data transmission. Therefore, the packet loss rate of the proposed REGPRJPC technique is significantly minimized by $28 \%$ and $39 \%$ when compared to existing methods. This confirms that reliability of REGPRJPC and reducing packet loss rate.

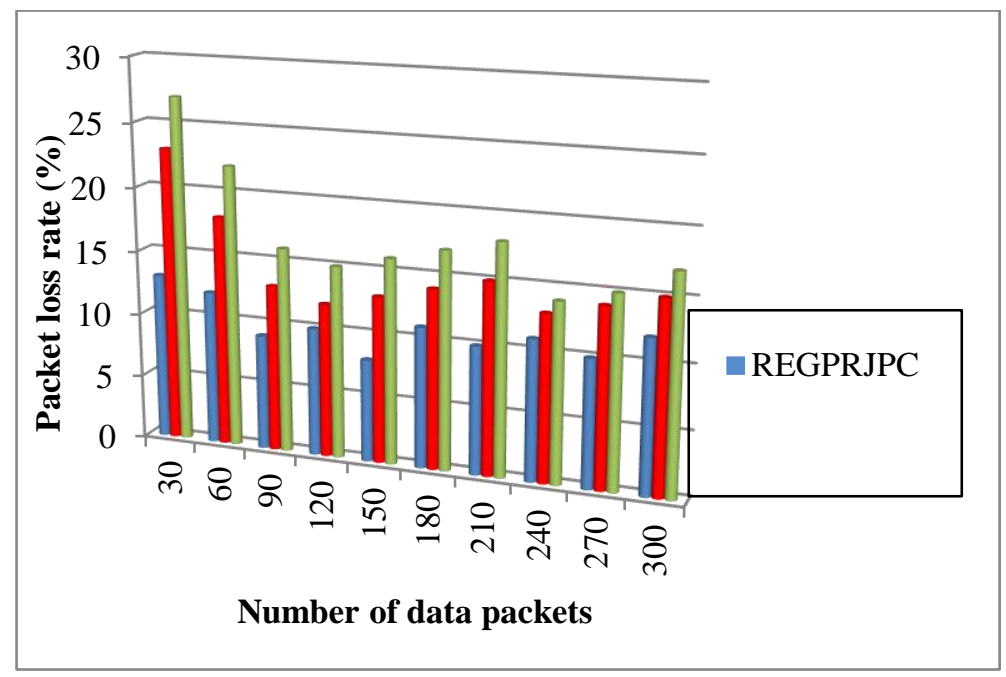

Figure 8. graphical illustration of packet loss rate

Results of packet loss rate are illustrated in figure 8 for three different methods namely the proposed REGPRJPC technique compared with existing PEERP [1] and efficient data transfer mechanism [2]. From figure 4, packet loss rate of REGPRJPC technique outperforms well in 
terms of achieving a lesser packet loss rate during the patient data transmission. This is because of REGPRJPC technique finds the energy-efficient node for transferring the data from source to sink. Moreover, higher bandwidth availability of SN enhances data delivery and lessen data loss during the patient data transmission.

\section{c. Impact of Throughput}

The throughput is referred to as an amount of patient data i.e. data packets are successfully delivered at the given time. The throughput is formulated as given below,

$$
T=\left(\frac{D P D}{t}\right) * 100
$$

Where $T$ denotes a throughput, $D P D$ represents the amount of data packet delivered, $t$ indicates time in sec. It is measured in bits per second (bps).

Table 4. Tabulation for throughput

\begin{tabular}{|c|c|c|c|}
\hline \multirow{2}{*}{$\begin{array}{c}\text { Size of the } \\
\text { data packet } \\
(\text { KB) }\end{array}$} & REGPRJPC & PEERP & $\begin{array}{c}\text { Efficient data } \\
\text { transfer } \\
\text { mechanism }\end{array}$ \\
\cline { 2 - 4 } & 165 & 152 & 145 \\
\hline 20 & 180 & 165 & 155 \\
\hline 40 & 215 & 198 & 182 \\
\hline 60 & 243 & 225 & 199 \\
\hline 80 & 265 & 240 & 223 \\
\hline 100 & 290 & 260 & 243 \\
\hline 120 & 312 & 290 & 270 \\
\hline 140 & 348 & 325 & 310 \\
\hline 160 & 450 & 410 & 386 \\
\hline 180 & 512 & 496 & 472 \\
\hline 200 & & & \\
\hline
\end{tabular}

Table 4 reports simulation analysis of throughput versus the size of data packets. Size of data packet is varied from $20 \mathrm{~KB}$ to $-200 \mathrm{~KB}$. According to the obtained results, the proposed REGPRJPC technique delivers higher sizes of the data in a given period. Let us consider $20 K B$ size of data packets considered and it is transmitted from the source node. By applying the REGPRJPC technique, 165 bits of the data packets are delivered to the sink. Whereas by applying the PEERP [1] and efficient data transfer mechanism [2], the 152 bits, and 145 bits of data packets successfully received in sink node. Various results of throughput using three methods are compared. The REGPRJPC enhances throughput by $13 \%$ and $23 \%$ as compared to [1] and [2]. 


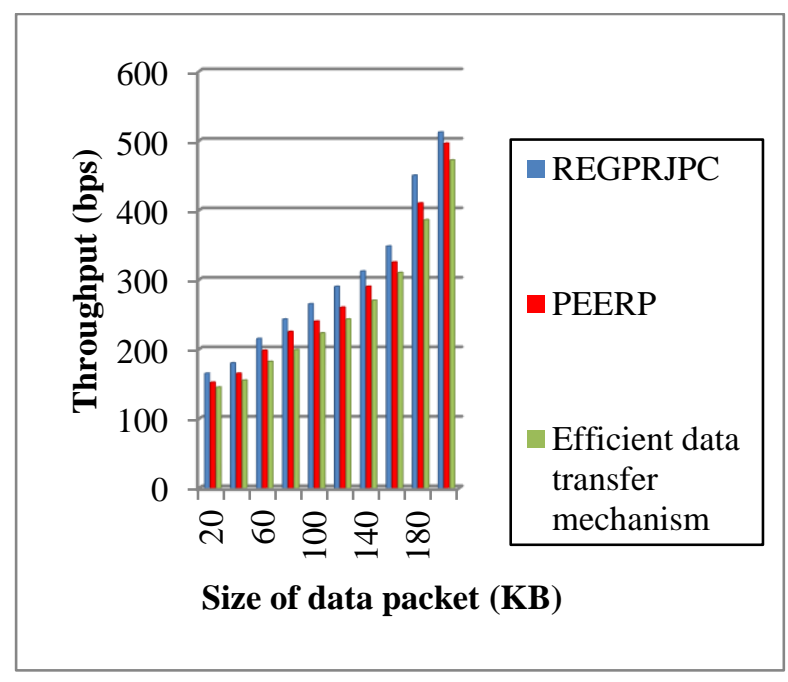

Figure 9. Graphical illustration of throughput

Figure 9 depicts the impact of throughput versus the different sizes of the data packets in the range of $20 \mathrm{~KB}-200 \mathrm{~KB}$ using three methods namely the REGPRJPC technique compared with existing PEERP [1] and efficient data transfer mechanism [2]. As depicted in figure 9, the proposed REGPRJPC technique provides better performance of achieving higher as compared to conventional data transfer scheme. Besides, while increasing the size of data packets, the throughput of data transmission is increased in three methods. But, throughput is higher using the proposed REGPRJPC technique. This is owing to the application of clustering-based data transmission. The Gaussian process regression function analyzes the SN to find the resourceefficient node for further processing in WSN.

\section{d. impact of an end to end delay}

End-to-End Delay is measured as amount of time-delayed to deliver data packets from source to sink. The delay is measured as difference among data packet arrival time and data packet sending time. Delay is formulated as given below,

$$
E_{D}=\text { time }_{a r}-\text { time }_{s d}
$$

Where, $E_{D}$ denotes end to end delay, time $_{a r}$ symbolizes data packet arrival time, $D P_{s d}$ indicates data packet sending time. It is measured in milliseconds (ms).

Table 5. Tabulation for end to end delay

\begin{tabular}{|c|c|c|c|}
\hline \multirow{2}{*}{$\begin{array}{c}\text { Number of sensor } \\
\text { nodes }\end{array}$} & REGPRJPC & PEERP & $\begin{array}{c}\text { Efficient data } \\
\text { transfer } \\
\text { mechanism }\end{array}$ \\
\cline { 2 - 4 } & 16 & 18 & 20 \\
\hline $\mathbf{5 0}$ & 19 & 22 & 24 \\
\hline $\mathbf{1 0 0}$ & 20 & 24 & 26 \\
\hline $\mathbf{1 5 0}$ & 22 & 25 & 28 \\
\hline $\mathbf{2 0 0}$ & 23 & 26 & 29 \\
\hline $\mathbf{2 5 0}$ & & &
\end{tabular}




\begin{tabular}{|l|l|l|l|}
\hline $\mathbf{3 0 0}$ & 25 & 28 & 30 \\
\hline $\mathbf{3 5 0}$ & 27 & 30 & 32 \\
\hline $\mathbf{4 0 0}$ & 29 & 31 & 33 \\
\hline $\mathbf{4 5 0}$ & 31 & 33 & 35 \\
\hline $\mathbf{5 0 0}$ & 33 & 35 & 38 \\
\hline
\end{tabular}

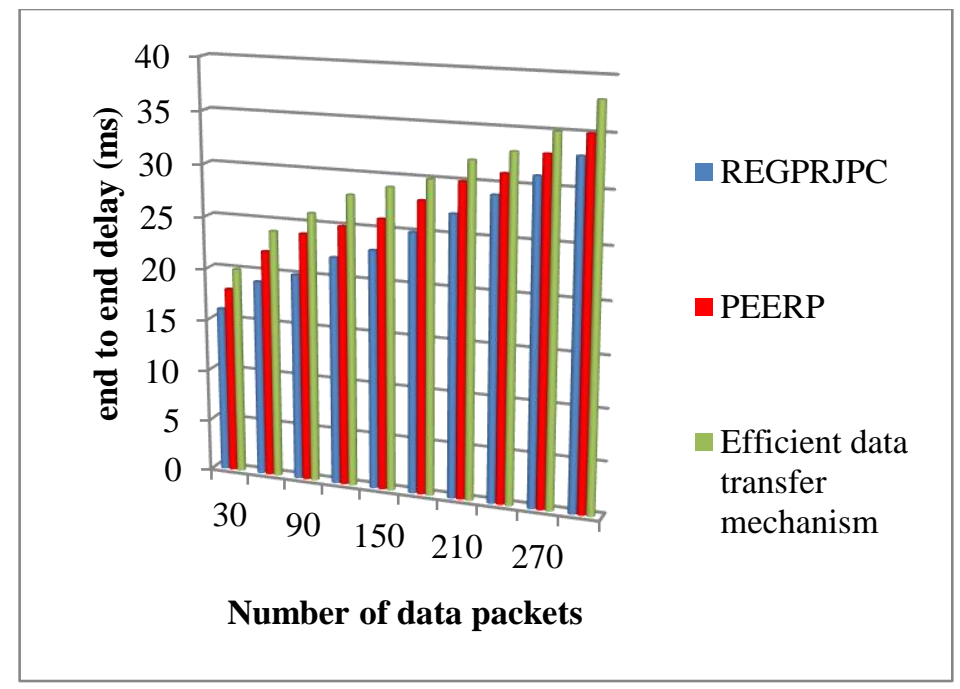

Figure 10. Graphical illustration of end-to-end delay

Table 5 and figure 10 illustrate the simulation results of delay. The observed results indicate that REGPRJPC technique offers superior performance than conventional methods. As shown in figure 8 , the delay of the three methods is increased while increasing the number of data packets (i.e. patient data). But comparatively the REGPRJPC technique minimizes the delay during the patient data transmission. Main reason for this improvement is $\mathrm{CH}$ selection-based data transmission instead of using many nodes. The source node transmits data to own $\mathrm{CH}$. Then $\mathrm{CH}$ discovers neighboring $\mathrm{CH}$ through the time of flight method. In this way, the patient data from source to sink is efficiently transmitted with minimum time.

Consider, number of nodes is 50, delay of REGPRJPC technique is $16 \mathrm{~ms}$ whereas delays of conventional methods PEERP [1] and efficient data transfer mechanism [2] are 18ms and $20 m s$. The observed numerical analysis proves that the proposed REGPRJPC technique outperforms well than the conventional methods. Similarly, the various results are obtained for different methods with. Delay of data transmission is reduced using REGPRJPC by $10 \%$ and $18 \%$ when compared to [1] and [2].

\section{Conclusion}

REGPRJPC technique is developed for resource-efficient reliable data transmission in Healthcare. At initial step, the entire network is partitioned into different clusters based on node residual energy and bandwidth consumption. The Jarvis Patrick clustering technique is applied to group the SN with the help of Gaussian process regression. The regression analyzes the SN along with the resource utilization. After that, the $\mathrm{CH}$ is chosen depends on higher residual energy and lesser bandwidth consumption to enhance data delivery. Then time of flight method is applied to find the neighboring $\mathrm{CH}$ for reliable data transmission from source to sink node. 
The simulation program is conducted to evaluate the efficiency of our REGPRJPC technique. Then the proposed REGPRJPC techniques are compared against conventional techniques. The results of REGPRJPC technique prolonged networks lifetime and improves the delivery ratio, throughput and lessen delay and loss rate.

\section{References}

[1] Ben othman soufienea, Abdullah Ali Bahatta, Abdelbasset Trad, Habib Youssef, "PEERP: An Priority-Based Energy-Efficient Routing Protocol for Reliable Data Transmission in Healthcare using the IoT", Procedia Computer Science, Volume 175, 2020, Pages 373-378

[2] Begum Seema, Nianmin Yao, Anil Carie \& Syed Bilal Hussain Shah, "Efficient data transfer in clustered IoT network with cooperative member nodes", Multimedia Tools and Applications, Springer, Volume 79, 2020, Pages 34241-34251

[3] Shuisheng Li, Baoxin Zhang, Panfeng Fei, P. Mohamed Shakeel, R. Dinesh Jackson Samuel, "Computational efficient wearable sensor network health monitoring system for sports athletics using IoT", Aggression and Violent Behavior, Elsevier, 2020, Pages 1-16

[4] Amir Abbas Baradaran and Keivan Navi, "HQCA-WSN: High-quality clustering algorithm and optimal cluster head selection using fuzzy logic in wireless sensor networks", Fuzzy Sets and Systems, Elsevier, Volume 389, 15 June 2020, Pages 114-144

[5] Amit Karmaker, Mohammad Shah Alam, Md. Mahedee Hasan Md., Andrew Craig, "An energy-efficient and balanced clustering approach for improving throughput of wireless sensor networks", International Journal of Communication System, Vol. 33, Issue 3, 2020, Pages 1-22

[6] Noureddine Moussa, Zakaria Hamidi-Alaoui \& Abdelbaki El Belrhiti El Alaoui, "ECRP: an energy-aware cluster-based routing protocol for wireless sensor networks", Wireless Networks, Springer, Volume 26, 2020, Pages 2915-2928

[7] Shalli Rani, Syed Hassan Ahmed \& Ravi Rastogi, "Dynamic clustering approach based on wireless sensor networks genetic algorithm for IoT applications", Wireless Networks, Springer, Volume 26, 2020, Pages 2307-2316

[8] Anurag Shukla \& Sarsij Tripathi, "A multi-tier based clustering framework for scalable and energy efficient WSN-assisted IoT network", Wireless Networks, Volume 26, 2020, 3471-3493.

[9] AmirSeyyedabbasi, Farzad Kiani, "MAP-ACO: An efficient protocol for multi-agent pathfinding in real-time WSN and decentralized IoT systems", Microprocessors and Microsystems, Elsevier, Volume 79, November 2020, 103325

[10] Antar Shaddad H.Abdul-Qawy, Nasr MusaedS. Almurisi, Srinivasulu Tadisetty, "Classification of Energy Saving Techniques for IoT-based Heterogeneous Wireless Nodes", Procedia Computer Science, Elsevier, Volume 171, 2020, Pages 2590-2599

[11] Om Jee Pandey and Rajesh M. Hegde, "Low-Latency and Energy-Balanced Data Transmission Over Cognitive Small World WSN", IEEE Transactions on Vehicular Technology, Volume 67, Issue 8, 2018, Pages $7719-7733$

[12] Yongnu Jin, Kyung Sup Kwak, Sang-Jo Yoo, "A Novel Energy Supply Strategy for Stable Sensor Data Delivery in Wireless Sensor Networks", IEEE Systems Journal, Volume 14, Issue 3, 2020, Pages 3418 - 3429

[13] Vijayakumar Singanamalla, Rizwan Patan , Mohammad S. Khan, Suresh Kallam, "Reliable and energy efficient emergency transmission in wireless sensor networks", Internet technology letter, Volume 2, Issue 2, 2019, Pages 1-6

[14] Sepehr Ebrahimi Mood \& Mohammad Masoud Javidi, "Energy-efficient clustering method for wireless sensor networks using modified gravitational search algorithm", Evolving Systems, Springer, Volume 11, 2020, Pages 575-587

[15] Zhidong Zhao, Duoshui Shi Guohua Hui, Xiaohong Zhang, "An Energy-Optimization Clustering Routing Protocol Based on Dynamic Hierarchical Clustering in 3D WSNs", IEEE Access, Volume 7, 2019, Pages $80159-80173$ 
[16] Deepak Mehta, Sharad Saxena, "MCH-EOR: Multi-objective Cluster Head Based Energy-aware Optimized Routing algorithm in Wireless Sensor Networks", Sustainable Computing: Informatics and Systems, Elsevier, Volume 28, 2020, Pages 1-34

[17] Kale Navnath Dattatraya, K. Raghava Rao, "Hybrid based Cluster Head Selection for Maximizing Network Lifetime and Energy Efficiency in WSN”, Journal of King Saud University - Computer and Information Sciences, Elsevier, 2019, Pages 1-16

[18] Hassan El Alami and Abdellah Najid, "ECH: An Enhanced Clustering Hierarchy Approach to Maximize Lifetime of Wireless Sensor Networks", IEEE Access, Volume 7, 2019, Pages 107142 - 107153

[19] Marlon Jeske, Valerio Rosset, Mari'a C.V. Nascimento, "Determining the Trade-offs Between Data Delivery and Energy Consumption in Large-scale WSNs by Multi-Objective Evolutionary", Computer Networks, Elsevier, Volume 179, 2020, Pages 1-31

[20] Aripriharta, Wang Zhi Hao, Muladi, Gwo-Jiun Horng, Gwo-Jia Jong, “A New Bio-Inspired for Cooperative Data Transmission of IoT”, IEEE Access, Volume 8, 2020, Pages 161884 - 161893. 